\title{
A vesicating dermatitis from a blister beetle, in the neck of Septuagenarian Saudi male
}

\author{
Khalid Al Aboud', Daifullah Al Aboud², Ahmad Al Aboud ${ }^{3}$
}

\begin{abstract}
${ }^{1}$ Department of Dermatology, King Faisal Hospital in Makkah, Makkah, Saudi Arabia, ${ }^{2}$ Department of Dermatology, Taif University, Taif, Saudi Arabia, ${ }^{3}$ Department of Dermatology, King Abdullah Medical City, Makkah, Saudi Arabia
\end{abstract}

Corresponding author: Dr. Khalid Al Aboud, E-mail: amoa65@hotmail.com

Sir,

A 72 years old Saudi male presented with an itchy and mildly painful skin lesion on the left side of the neck after being contacted before 2 days by a blister beetle while sleeping at night.

On examination, there is a well demarcated skin lesion, which has 'burnt' appearance, with streaks of hyperpigmentation at its lower border (Fig. 1).

The patient was able to identify the insect responsible, which is photographed in (Fig. 2).

He responded to topical steroid and an oral antihistamine.

The nature of this lesion and general instructions to avoid its recurrence were also given to the patient.

Blister beetle dermatitis is a sort of an irritant contact dermatitis. It is also known as dermatitis linearis, or paedrous dermatitis (PD). It is caused by chemical substances (Cantharidin, Pederin, Pseudopederin or Pederone), released from some beetles when crushed, pressed or come in contact with the skin [1-3]. The beetles do not bite or sting.

The lesion may take 1-2 days to develop but usually appear in the morning following a sleep.

As occurred in our case, the lesion usually affects the head and neck area.

There are approximately 250 known species of Paederus beetles. Most of these beetles contain vesicant fluid.

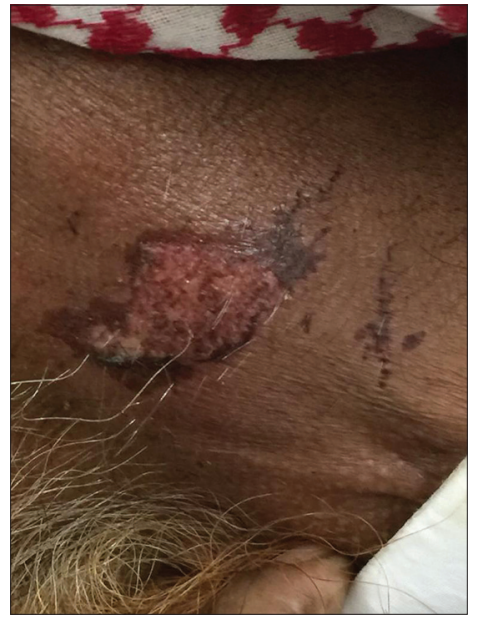

Figure 1: A 'burnt skin' appearance of the lesion with streaks of hyperpigmentation from dripping of irritant beetle fluid.

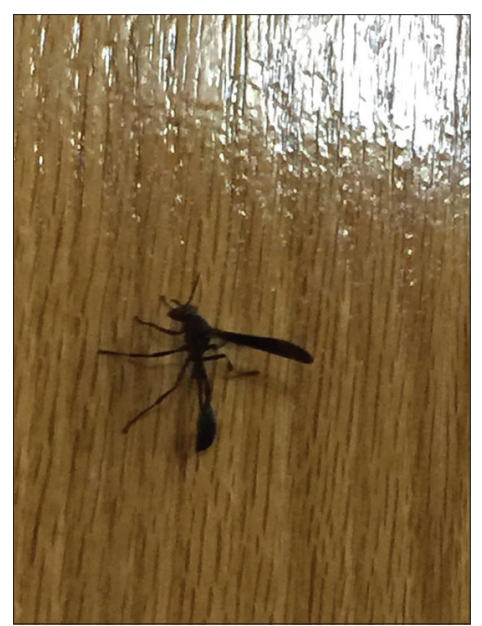

Figure 2: The type of beetle involved in the skin lesion.

Examples of species, belonging to genus Paederus, involved in PD include Paederus fuscipes, P. irritans, P. sabacus, and P. himalayicus.

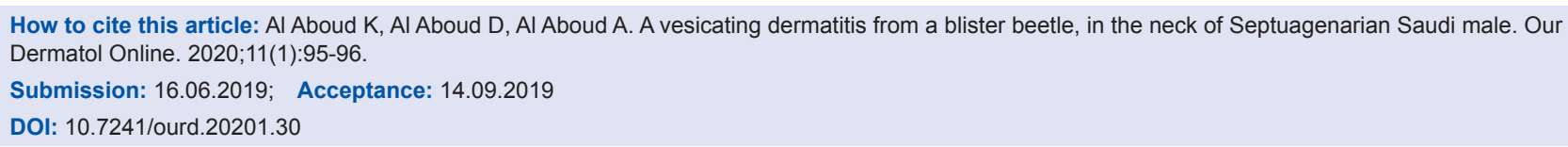


We could not have access to identify the exact species in this case, as there are no nearby entomological centers.

PD may cause extensive ulcerations but systemic symptoms do not usually occur.

Farmers and workers living outdoors are mostly at risk but people living indoor can be also affected.

The condition is not rare but seems to be under-reported. A series of 37 and 46 cases have been published [1,2].

PD takes a seasonal preference being maximum between May to July.

Tenderness of the affected area is thought to be an important clinical sign for PD.

With a proper history the diagnosis is not difficult but differential diagnoses may include burns by liquids, herpes simplex, herpes zoster, allergic or irritant contact dermatitis, bullous impetigo and phyto photo dermatitis.

Simple preventive measures can be undertaken based on the behavioral pattern of this nocturnal beetle which is known to breed in damp places, with high humidity.

Measures which were proved to be effective include;

- The housing of the workers in the farms is preferably not to be inside the agricultural land or near decaying garbage.
- Farmers are advised to use proper clothing or mosquito nets during the sleep.

- Regular spraying of the farms with insecticides such as a combination of Baygon (carbamate group) and Malathion (organophosphorus group).

- Avoiding resting in open areas close to paddy fields and neon or fluorescent lamps during night time, and proper use of insect repellants.

- Health education to the people about the condition and how to prevent it and the ways to deal with it.

\section{Consent}

The examination of the patient was conducted according to the Declaration of Helsinki principles.

\section{REFERENCES}

1. Srihari S, Kombettu AP, Rudrappa KG, Betkerur J. Paederus Dermatitis: A Case Series. Indian Dermatol Online J. 2017;8:361-4.

2. Verma CR, Agarwal S. Blistering Beetle Dermatitis: An Outbreak. Med J Armed Forces India. 2006;62:42-4.

3. Singh A, Singh AK, Singh D, Varghese A. Blister beetle dermatitis: few observations helping in diagnosis. Int J Prev Med. 2013;4:241.

Copyright by Khalid Al Aboud, et al. This is an open-access article distributed under the terms of the Creative Commons Attribution License, which permits unrestricted use, distribution, and reproduction in any medium, provided the original author and source are credited.

Source of Support: Nil, Conflict of Interest: None declared. 